ARTICLE

\title{
Transcription shapes genome-wide histone acetylation patterns
}

\author{
Benjamin J. E. Martin (D) ${ }^{1}$, Julie Brind'Amour (10 ${ }^{2}$, Anastasia Kuzmin ${ }^{1}$, Kristoffer N. Jensen², Zhen Cheng Liu', \\ Matthew Lorincz (D) $^{2} \&$ LeAnn J. Howe (i) ${ }^{1 \times}$
}

Histone acetylation is a ubiquitous hallmark of transcription, but whether the link between histone acetylation and transcription is causal or consequential has not been addressed. Using immunoblot and chromatin immunoprecipitation-sequencing in S. cerevisiae, here we show that the majority of histone acetylation is dependent on transcription. This dependency is partially explained by the requirement of RNA polymerase II (RNAPII) for the interaction of $\mathrm{H} 4$ histone acetyltransferases (HATs) with gene bodies. Our data also confirms the targeting of HATs by transcription activators, but interestingly, promoter-bound HATs are unable to acetylate histones in the absence of transcription. Indeed, HAT occupancy alone poorly predicts histone acetylation genome-wide, suggesting that HAT activity is regulated postrecruitment. Consistent with this, we show that histone acetylation increases at nucleosomes predicted to stall RNAPII, supporting the hypothesis that this modification is dependent on nucleosome disruption during transcription. Collectively, these data show that histone acetylation is a consequence of RNAPII promoting both the recruitment and activity of histone acetyltransferases.

\footnotetext{
${ }^{1}$ Department of Biochemistry and Molecular Biology, Life Sciences Institute, Molecular Epigenetics Group, University of British Columbia, 2350 Health Sciences Mall, Vancouver, BC, V6T 1 Z3 Canada. ${ }^{2}$ Department of Medical Genetics, Life Sciences Institute, Molecular Epigenetics Group, University of British Columbia, 2350 Health Sciences Mall, Vancouver, BC, V6T 1 Z3 Canada. ${ }^{\bowtie}$ email: ljhowe@mail.ubc.ca
} 
ysine acetylation of histone amino-terminal tails has been linked to gene expression for many decades ${ }^{1}$. More recently, genome-wide localization studies across eukaryotes, including yeast and mammals, revealed that histone tail acetylation primarily occurs at the promoters and $5^{\prime}$ ends of transcribed genes $^{2}$. Although some forms of acetylation have been referred to as "global" and "non-targeted" 3 , genome-wide occupancy studies show that histone acetylation levels correlate strongly with transcription, suggesting a causal relationship between the two.

Histone acetylation is catalyzed by conserved histone acetyltransferases (HATs), generally consisting of a catalytic subunit complexed with auxiliary proteins required for enzymatic activity and targeting ${ }^{4}$. Most HAT complexes have relatively low substrate specificity and modify multiple lysine residues within either $\mathrm{H} 3$ or H4. Thus, histone acetylation sites within $\mathrm{H} 3$ and $\mathrm{H} 4$ generally show similar distributions ${ }^{2}$, and mutations of histone lysine residues, with the exception of $\mathrm{H} 4 \mathrm{~K} 16$, result in comparable changes in gene expression ${ }^{5}$. Histone acetylation is a dynamic mark due to the activity of histone deacetylase complexes (HDACs). Similar to HATs, HDACs generally exist as multi-protein complexes with catalytic subunits that can deacetylate multiple lysines on one or more histones ${ }^{6}$.

In Saccharomyces cerevisiae, the most well-characterized proteins with lysine acetyltransferase activity are Gcn5 and Esa1, which are the catalytic subunits of multiple HAT complexes, including the H3-specific HATs, SAGA, and ADA for Gcn5, and the H4-specific HATs, NuA4, and Piccolo for Esa ${ }^{4}$. SAGA and NuA4 are targeted to gene promoters via an interaction between a shared subunit, Tra1, and DNA-bound transcription activators $^{7-9}$, which is thought to target acetylation of nucleosomes flanking promoters. This, together with the observation that Gcn 5 and Esa1 are required for transcription of multiple genes $^{10-13}$, has led to the widely accepted model that histone acetylation acts upstream of transcription initiation. It should be noted however that, in addition to histones, HATs acetylate many non-histone proteins involved in transcription initiation ${ }^{14}$. As such, whether SAGA and NuA4 activate transcription primarily through acetylation of core histones remains uncertain.

While the current model for targeting HATs by transcription activators upstream of transcription is widely accepted, there are several examples of histone acetylation being deposited as a consequence of transcription. Numerous HATs interact with co-transcriptional H3K4me $3^{15-18}$ and the phosphorylated carboxy-terminal domain (CTD) of RNAPII ${ }^{12,19}$. In addition, cotranscriptional histone exchange mediates incorporation of acetylated histones into nucleosomes within transcribed regions $^{20}$, and recent work has shown that RNA can promote the activity of CBP at enhancers ${ }^{21}$. Taken together these observations suggest that histone acetylation can also be a consequence of the transcription process. However, the relative contribution of these pathways to histone acetylation patterns remains unknown.

In this study, we sought to determine the relative contributions of the "causal" vs. "consequential" pathways for targeting histone acetylation to transcribed genes. We first found that inhibition of transcription results in rapid histone deacetylation in both yeast and mouse embryonic stem cells (mESCs), demonstrating that a significant portion of histone acetylation is a consequence of transcription. Loss of RNAPII also results in depletion of Epl1, a subunit of the yeast NuA4 and Piccolo HATs, from gene bodies, consistent with HAT targeting by RNAPII. Residual, transcription-independent Epl1 binding was observed over promoter regions, in agreement with the targeting of HATs by transcription activators, but surprisingly, acetylation in these regions is still transcription-dependent. Thus, although these results are consistent with previous models for targeting HATs, we found that HAT recruitment alone is insufficient to mediate acetylation of the associated nucleosomes, indicative of postrecruitment regulation of acetyltransferase activity. One model that is consistent with our data is that the acetylation of histones is dependent on nucleosome disruption by RNAPII. In agreement with this, we see increased acetylation at nucleosomes predicted to impede RNAPII passage.

\section{Results}

Histone acetylation is dependent on transcription. Despite the well-known correlation between histone acetylation and transcription, whether this posttranslational modification (PTM) is primarily a cause or consequence of transcription has not been definitively tested. We, therefore, sought to assess the dependence of histone acetylation on transcription by inhibiting RNAPII activity in S. cerevisiae. Previous studies have used the rpb1-1 temperature-sensitive mutant to disrupt transcription ${ }^{22}$. However, more recent experiments have suggested that this mutant does not directly inhibit RNAPII, as shifting the mutant to the restrictive temperature has minimal effects on transcript synthesis $^{23}$ and does not lead to rapid dissociation of RNAPII from gene bodies ${ }^{24}$. To achieve effective inhibition of RNAPII, we, therefore, treated cells with 1,10 phenanthroline monohydrate $(1,10-p t)$, which has been shown to rapidly inhibit transcript synthesis ${ }^{23}$. Confirming efficient transcription inhibition by 1,10 pt, we observed a global loss of RNAPII serine 5 CTD phosphorylation by immunoblot analysis (Fig. 1a) and rapid alterations in RNAPII distribution as determined by ChIP-seq (Fig. 1b, ChIP inputs shown in Supplementary Fig. 1a). Immunoblot analysis of yeast whole-cell extracts showed that within $15 \mathrm{~m}$ of transcription inhibition, a broad range of $\mathrm{H} 3$ and $\mathrm{H} 4$ acetylation marks were rapidly lost (Fig. 1a, c). Similar deacetylation was observed following, treatment with the transcription inhibitor thiolutin (Supplementary Fig. 2a), or degradation of Rpb2, the second largest subunit of RNAPII, using an auxin-inducible degron (Supplementary Fig. 2b). Notably, loss of acetylation was dependent on the histone deacetylases Rpd3 and Hda1 (Supplementary Fig. 2c) and could be almost completely blocked by prior treatment with the HDAC inhibitor TSA (Supplementary Fig. 2d), confirming active deacetylation upon transcription inhibition. Histone acetylation loss was due to disruption of HAT activity, rather than increased HDAC activity, as incubation with TSA following 1,10-pt treatment failed to restore histone acetylation (Supplementary Fig. 2e). HATs are conserved throughout Eukaryota and thus it is likely that acetylation is dependent on transcription in other organisms. Indeed, we found that inhibition of transcription by actinomycin $\mathrm{D}$, which inhibits transcription initiation, as indicated by loss of Ser5p (Fig. 1d), and transcription elongation ${ }^{25}$ in mESCs resulted in the loss of $\mathrm{H} 3 \mathrm{~K} 9 \mathrm{ac}$ and H3K27ac in bulk histones (Fig. 1d, e).

To confirm that histone acetylation loss is a direct consequence of transcription inhibition, we treated $S$. cerevisiae cells with 1,10 pt for $15 \mathrm{~m}$ and performed ChIP-seq for H3K23ac, H4K8ac, and H4K12ac. Consistent with previous studies ${ }^{26,27}$, we used nontranscribed regions to account for global changes in ChIP-seq experiments (see "Methods"). While no major changes to nucleosome occupancy or position were observed following the short transcription inhibition performed here, large decreases in histone acetylation were observed (Fig. 1f). Importantly, heatmaps of $\log _{2}$ fold changes in ChIP-seq signal upon 1,10-pt treatment showed that patterns of histone acetylation loss mirrored those of RNAPII (Supplementary Fig. 3a). In addition, histone deacetylation was limited to nucleosomes that lost RNAPII upon 1,10-pt treatment (Supplementary Fig. 3b). In contrast, regions with more stable RNAPII, including the $3^{\prime}$ ends of genes, showed slight increases in histone acetylation (Fig. 1f, 
a
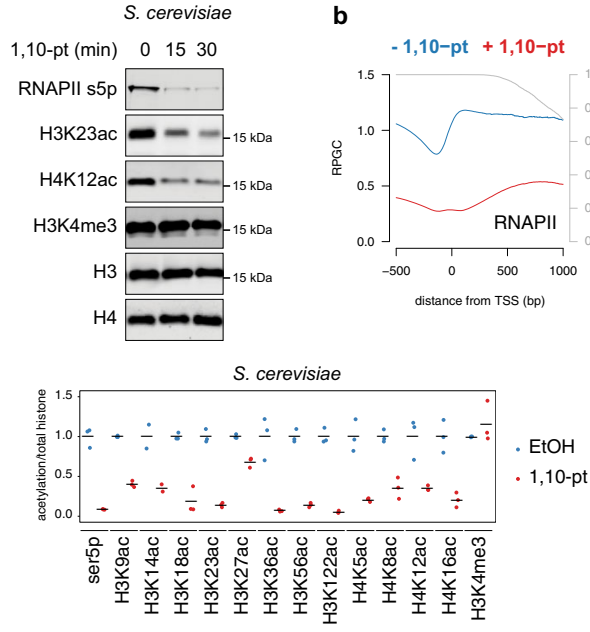

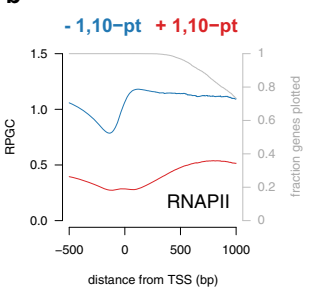

d

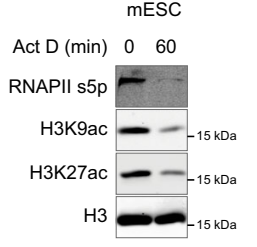

f
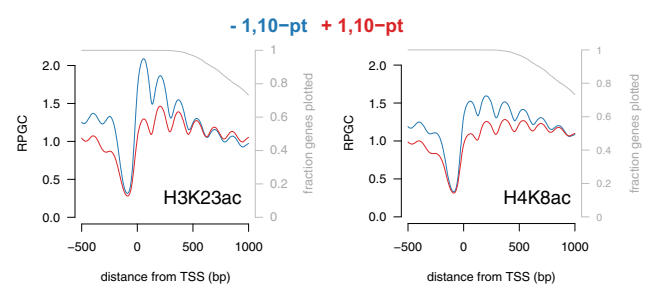

e

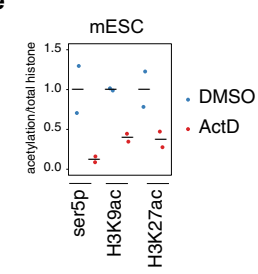

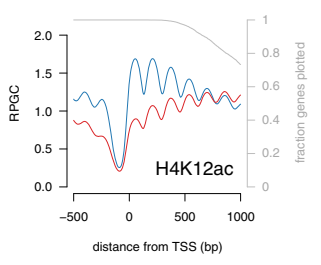

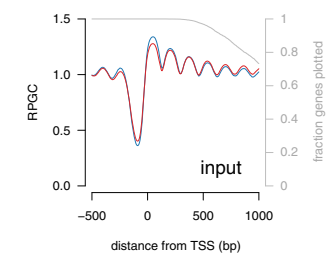

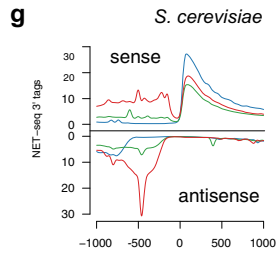

distance from the TSS (bp)

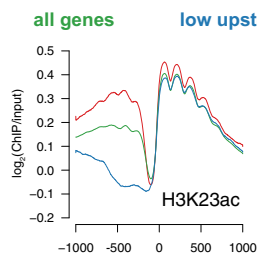

distance from the TSS (bp)

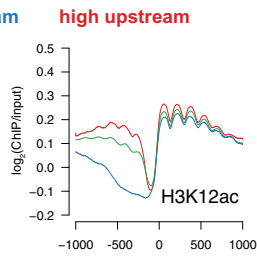

distance from the TSS (bp)

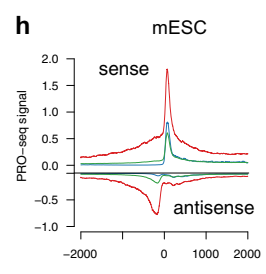

distance from the TSS (bo)
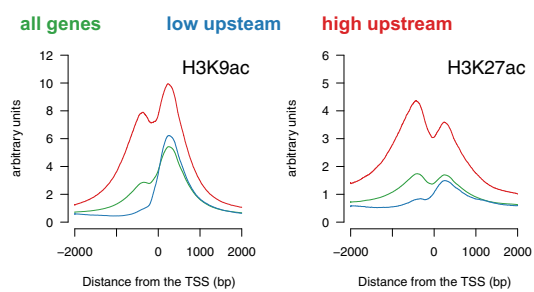

Fig. 1 The majority of histone acetylation is dependent on transcription. a Whole-cell extracts from S. cerevisiae cells before and after treatment with 1,10pt were subjected to immunoblot analysis with the indicated antibodies. Experiments were performed in triplicate with quantified results shown in (c). Raw immunoblot data is provided in the Source Data file. b Average profile of RNAPII (Rpb3 ChIP-seq ${ }^{73}$ ) at 5206 transcribed genes aligned by the TSS before (blue) and after a $15 \mathrm{~m}$ treatment with 1,10-pt (red). Only data until the polyadenylation site (PAS) was included, and the gray line represents the fraction of genes still being plotted. RPGC reads per genomic coverage, TSS transcription start site. c Strip plots of histone PTM immunoblot signals normalized to histone $\mathrm{H} 4$ levels from three independent yeast whole-cell extracts from cultures without (blue) and with (red) a 15-min treatment with 1,10-pt. Horizontal lines indicate the mean, with the vehicle control set to 1. d Nuclear extracts from mouse ESCs before and after treatment with actinomycin D were subjected to immunoblot analysis with the indicated antibodies. Experiments were performed in duplicate with quantified results shown in (e). Raw immunoblot data is provided in the Source Data file. e Strip plots of histone acetylation immunoblot signals normalized to histone H3 from two mESC nuclear extracts from independent cultures without (blue) and with (red) actinomycin D. Horizontal lines indicate the mean, with the vehicle control set to 1. f Average profile of H3K23ac, H4K8ac, and H4K12ac (MNase ChIP-seq) and input at 5206 genes aligned by the TSS before (blue) and after (red) a 15min treatment with 1,10-pt. Data from 1,10-pt-treated cells was normalized to untreated (see "Methods"). $\mathbf{g}$ The average signal relative to the TSS for $S$. cerevisiae NET-seq $41, \mathrm{H} 3 \mathrm{~K} 23 \mathrm{ac}^{33}$, and H4K12ac ChIP-seq data relative to all genes (green) or 832 transcribed genes that have low (blue) or high (red) upstream NET-seq signal. The ChIP data is presented as $\log _{2}(\mathrm{ChIP} /$ input) as nucleosome density is not consistent between the different gene bins. $\mathbf{h}$ The average signal relative to the TSS for PRO-seq ${ }^{74}$, and H3K9ac and H3K27ac ChIP-seq data relative to all genes (green) or 3035 transcribed genes that have low (blue) or high (red) upstream PRO-seq signal.

Supplementary Fig. 3a, b). While this may result from enhanced targeting of HATs displaced from other loci, we cannot rule out the possibility that our scaling approach did not fully account for global decreases in histone acetylation. Irrespective, we find that $\mathrm{H} 3 \mathrm{~K} 23 \mathrm{ac}, \mathrm{H} 4 \mathrm{~K} 8 \mathrm{ac}$, and $\mathrm{H} 4 \mathrm{~K} 12 \mathrm{ac}$ were primarily deacetylated at regions that lost RNAPII upon transcription inhibition, suggestive of a direct effect. Collectively these results demonstrate that a large portion of histone acetylation is a consequence of transcription, which is inconsistent with the prevalent model that histone acetylation is primarily targeted to active genes upstream of transcription. Although this result was initially surprising, it is consistent with reports demonstrating that histone acetylation upstream of promoters is limited to those with divergent transcription ${ }^{28-30}$, which was confirmed by analyses of data from both yeast (Fig. 1g, Supplementary Fig. 4) and mESCs (Fig. 1h).

Piccolo is targeted by RNAPII. The simplest explanation for the transcription dependence of histone acetylation is that RNAPII targets HATs to transcribed genes. Indeed, previous work has shown that genome-wide occupancies of Gcn5, Sas3, and Epl1, a common subunit of Esal-dependent $\mathrm{HATs}^{31}$, are increased at highly transcribed genes ${ }^{27,32,33}$. To directly test this, we performed ChIP-seq for Epl1 prior to and following $15 \mathrm{~m}$ of transcription inhibition. In the absence of 1,10-pt, Epl1 was enriched over gene bodies and depleted immediately upstream of TSSs (Fig. 2a, inputs shown in Supplementary Fig. 1b). Following transcription inhibition, Epll occupancy over the $5^{\prime}$ genic regions diminished significantly, mirroring RNAPII loss in these regions (compare Figs. 2a and 1b, Supplementary Fig. 5a, b). In contrast, Epl1 bound upstream of TSSs was less sensitive to transcription inhibition (Fig. 2a), and analysis of genes lacking divergent transcription to avoid signal of Epl1 on upstream gene bodies, showed a peak of Epll binding $400 \mathrm{bp}$ upstream of TSSs in both actively transcribing and transcription-inhibited cells (Fig. 2b, inputs shown in Supplementary Fig. 1c). Collectively, these results suggest that Epl1 is recruited to chromatin through two pathways: transcription-dependent targeting to gene bodies and transcription-independent binding upstream of TSSs.

Epl1 is a component of two HATs: NuA4 and Piccolo ${ }^{31}$. Both complexes contain a HAT module consisting of Esa1, Epl1, and Yng2, but only NuA4 contains Tra1, which mediates the interaction of this HAT with transcription activators ${ }^{7,9}$. Deletion 
a

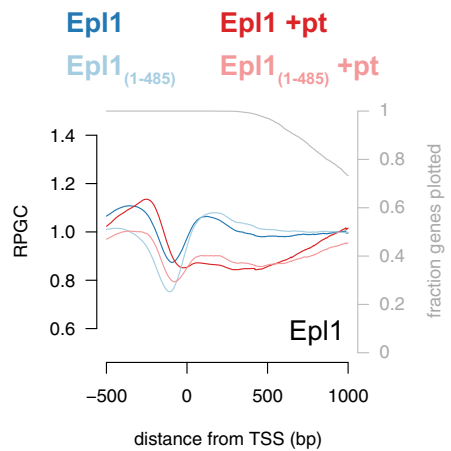

b

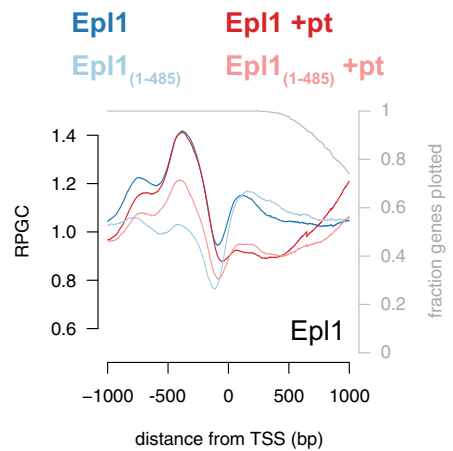

d

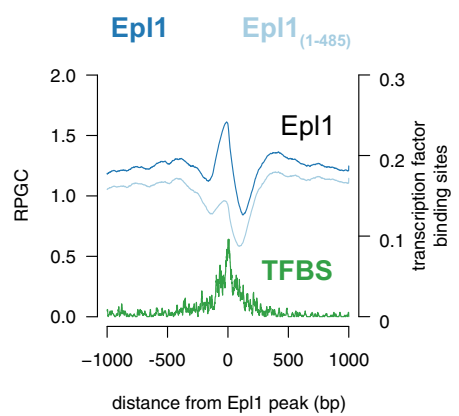

e
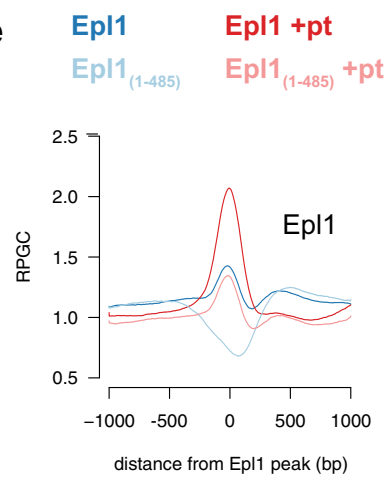

h

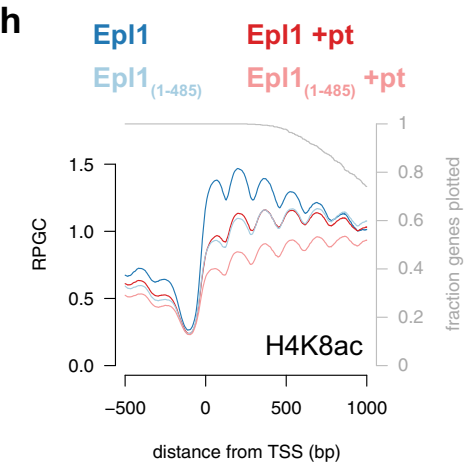

$\mathbf{f}$

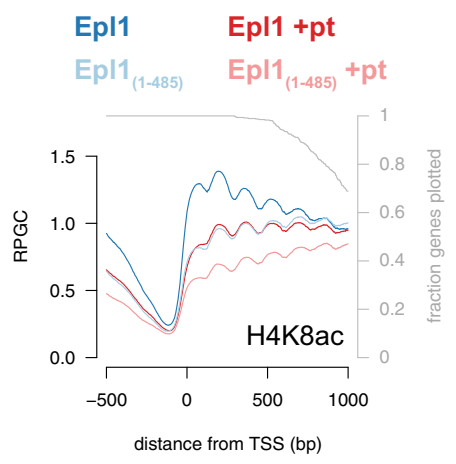

g

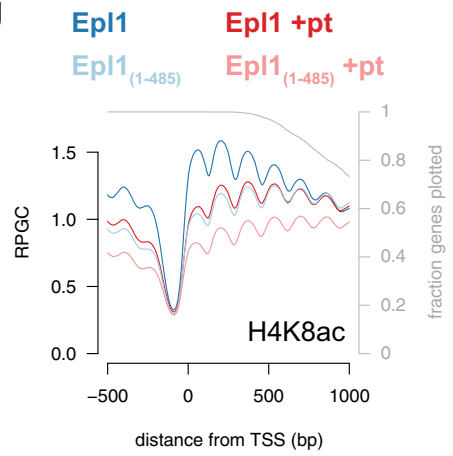

- EtOH

- 5 min post-1,10-pt removal

- 30 min post-1,10-pt removal

Fig. 2 Transcription promotes the interaction of H4-specific HATs with chromatin. a, b Average profile of Epl1 (dark lines) or Epl1 $1-485$ (light lines) ChIPseq from sonicated extracts at all genes (5206) (a) or 832 unidirectional promoter genes (b) aligned by the TSS before (blue) and after (red) a 15-min treatment with 1,10-pt. Only data until the PAS was included, and the gray line represents the fraction of genes plotted for each position. Data from drugtreated and mutant cells were normalized to untreated wild-type (see "Methods"). RPGC reads per genomic coverage, TSS transcription start site. c Strip plots of histone acetylation immunoblot signals normalized to histone $\mathrm{H} 4$ levels from two independent yeast whole-cell extracts. Wild type and set1 $\Delta$ cells were either untreated (red) or treated with 1,10-pt for $30 \mathrm{~m}$, followed by TSA treatment for an additional $30 \mathrm{~m}$ (orange), before being washed into fresh media containing TSA. Samples were collected 5 (green) and 30 (blue) minutes post wash. Horizontal lines indicate the mean. d Average profile of Epl1 (dark blue) and Epl1 $1-485$ (light blue) ChIP-seq from MNase-treated chromatin relative to the center of 562 regions showing strong Epl1 peaks (peak coordinates are provided in the Source Data file). The abundance of transcription factor binding sites (TFBS) ${ }^{38}$ across the region is shown in green. e Average profile of Epl1 (dark lines) and Epl1 $1_{-485}$ (light lines) ChIP-seq from sonicated extracts from cells before (blue) and after (red) a 15-min treatment with 1,10-pt, relative to the center of 562 regions showing strong Epl1 peaks. f-h Average profiles of H4K8ac ChIP-seq from MNase-digested extracts from Epl1 (dark lines) and Epl1 $1-485$ (light lines)-expressing strains, before (blue) and after (red) a 15-min treatment with 1,10-pt, at genes with strong Epl1 peaks (f), all genes ( $\mathbf{g}$ ), or 832 genes with promoters lacking divergent transcription (h).

of the 348 C-terminal amino acids of Epl1 (Epl1 $\left.1_{1-485}\right)$, disrupts the incorporation of the HAT module into NuA4 ${ }^{31}$, and thus cells expressing Epl1 $1_{1-485}$ are thought to have Piccolo, but not NuA4. We used this information to determine whether bound Epll was in the form of $\mathrm{NuA} 4$ or Piccolo by repeating ChIP-seq in cells expressing Epl1 $1_{1-485}$. Analysis of all and just unidirectional promoters (Fig. 2a, b) showed that truncation of Epl1 resulted in a loss of binding upstream of TSSs, with little change over gene bodies, which is consistent with Piccolo and NuA4 binding gene bodies and promoters respectively.

RNAPII-dependent targeting of Esal to gene bodies was proposed to occur through recognition of $\mathrm{H} 3 \mathrm{~K} 4$ methylation by the PHD finger of Yng2 ${ }^{16,34,35}$. However, Fig. 1a shows that $\mathrm{H} 3 \mathrm{~K} 4 \mathrm{me} 3$ was resistant to transcription inhibition, indicating that acetylation loss was not due to the removal of this PTM. Moreover, genome-wide analysis of HAT occupancy suggests 
that, while $\mathrm{H} 3 \mathrm{~K} 4$ methylation can promote HAT processivity, it is dispensable for targeting ${ }^{27}$. To determine whether $\mathrm{H} 3 \mathrm{~K} 4$ methylation is important for transcription-dependent acetylation, wild-type and set $1 \Delta$ strains were treated with 1,10-pt, which was then washed out to allow cells to resume transcription prior to the assessment of acetylation levels by immunoblot. Figure $2 \mathrm{c}$ shows that acetylation was restored with similar kinetics in wild-type and mutant strains. Thus, the ability of Piccolo to acetylate bulk nucleosomes was not dependent on the presence of $\mathrm{H} 3 \mathrm{~K} 4$ methylation following transcription resumption. It should be noted, however, that because we analyzed bulk histones, we cannot rule out the possibility that loss of $\mathrm{H} 3$ methylation altered the ability of Esa1 to acetylate nucleosomes at specific regions, but this loss is masked by increased acetylation in other regions.

Previous work has also implicated RNAPII serine 5 phosphorylation and H3K36 methylation in targeting NuA4 to gene bodies $^{12,35,36}$. While this is inconsistent with our genome-wide data showing that disruption of NuA4 has minimal impact on Epl1 binding to these regions, using set2 $\Delta$ (Supplementary Fig. 6a) and analog-sensitive KIN28 (Supplementary Fig. 6b, c) mutants, we show that global histone acetylation was not dependent on H3K36 methylation nor RNAPII serine 5 phosphorylation by Kin28, either alone or in combination with loss of H3K4 methylation. Thus, although the exact mechanism for targeting Epll to gene bodies remains to be identified, these results show that the transcription-dependence of histone $\mathrm{H} 4$ acetylation can be partially explained by a requirement of transcription for targeting Piccolo to gene bodies.

NuA4 is targeted by transcription activators. To confirm that Epll bound upstream of TSSs represents activator targeted HAT complex, we sought to improve the resolution of our Epl1 ChIPseq data. To this end, we repeated Epll ChIP-seq in actively transcribing cells using chromatin fragmented by micrococcal nuclease, which was previously shown to detect nonhistone protein complexes bound to $\mathrm{DNA}^{37}$. Epl1-bound, MNaseresistant DNA showed a similar pattern of genome-wide localization as input chromatin (Supplementary Fig. 1d, e), although Epl1 and Epl1 $1_{(1-485)}$, but not untagged ChIP-sequence fragments, showed increased abundance on highly expressed genes (Supplementary Fig. S1f-h). Using a high stringency cut-off (see methods), we identified 562 promoters with strong peaks of Epl1 (Fig. 2d, inputs shown in Supplementary Fig. 1i). These peaks originated from sub-nucleosome sized DNA fragments that did not precipitate with anti-acetyl-histone antibodies (Supplementary Fig. 7) and thus were unlikely to represent nucleosomes. NDRs with Epl1 peaks were wider (mean of 297 vs. $164 \mathrm{bp}, p$ value of two-sided students $t$ test $\left.2.0 \times 10^{-49}\right)$ and associated with more highly expressed genes ( $p$ value of two-sided students $t$ test a

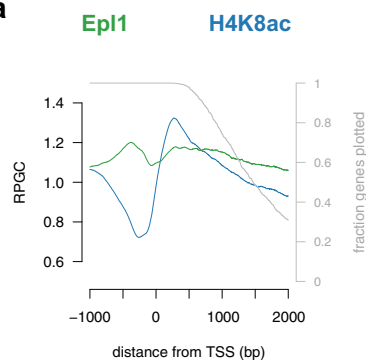

e
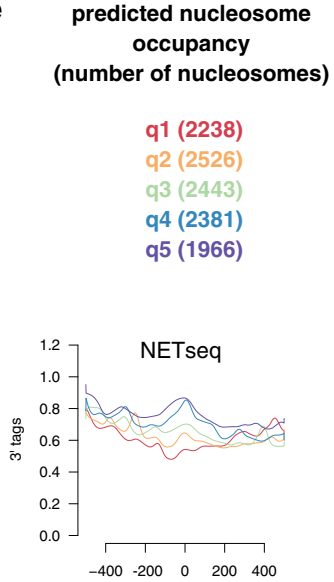

distance from dyad (bp) b $\quad$ Epl1 H4K12ac
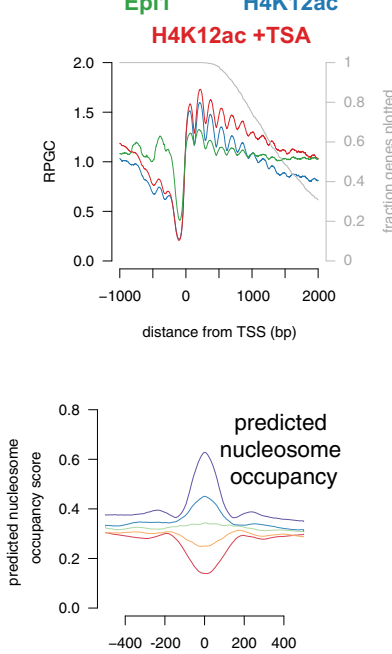

distance from dyad (bp)

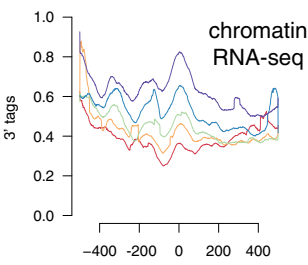

distance from dyad (bp) c
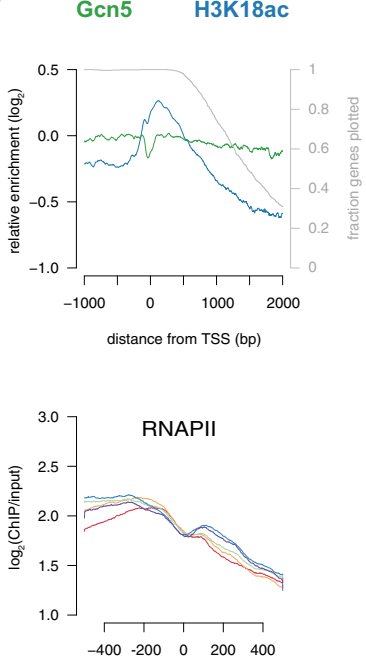

distance from dyad (bp)

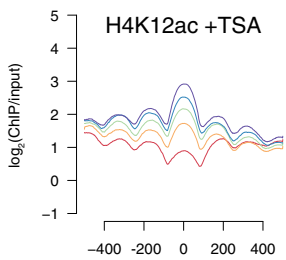

distance from dyad (bp) d
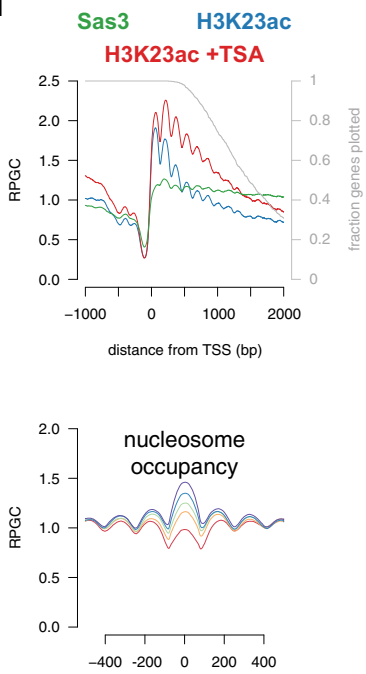

distance from dyad (bp)

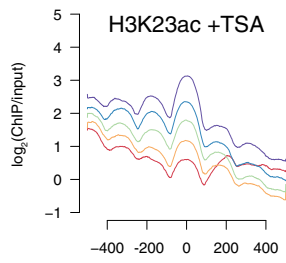

distance from dyad (bp)

Fig. 3 The activity of histone acetyltransferases are regulated post-recruitment. a, b Average profiles of Epl1 (green) and H4 acetylation (blue) ChIP from sonicated 27 (a) or MNase-digested chromatin (b) at 832 unidirectional promoter genes aligned by the TSS. Only data until the PAS was included, and the gray line represents the fraction of genes plotted for each position. Data from TSA-treated cells (red) was normalized to untreated wild-type (see "Methods"). RPGC reads per genomic coverage, TSS transcription start site. c, d Average profiles of Gcn5 (green) and H3K18ac (blue) ChIP75 from sonicated chromatin (c) and Sas3 (green) and H3K23ac (blue) ChIP from MNase-digested chromatin (d) at 832 unidirectional promoter genes aligned by the TSS. e Genic nucleosomes 47 in the middle quintile for RNAPII occupancy [ $\log _{2}$ (Rpb3/input) 50 bp upstream and downstream of the nucleosome dyad] were divided into quintiles based on predicted nucleosome occupancy 48 over the same region. Shown are profiles upstream and downstream of the nucleosome dyad for predicted nucleosome occupancy, RNAPII (Rpb3 ChIP-seq ${ }^{73}$ ), nucleosome occupancy (MNase-seq), NET-seq ${ }^{41}$, chromatin RNAseq $^{41}$, and nucleosome-normalized H4K12ac and H3K23ac ${ }^{33}$ ChIP-seq from TSA-treated cells for the first (red), second (orange), third (green), fourth (blue), fifth (purple) quintiles for predicted nucleosome occupancy. 
$3.5 \times 10^{-70}$ ) than all NDRs. Importantly, these peaks overlapped regions with transcription factor binding sites ${ }^{38}$, and Epll occupancy in these regions was reduced following Epl1 truncation (Fig. 2d), consistent with NuA4 targeting by transcription activators. A comparison of NDRs with Epl1 peaks to NDRs depleted for Epl1 signal revealed strong enrichment of DNA binding motifs for several transcription factors (Supplementary Fig. 8a). For the two top hits, Rap1 and Aft2, the DNA binding motifs were enriched at and immediately upstream of the Epl1 peak centers (Supplementary Fig. 8b, c), supporting a model of direct recruitment of Epl1, and consistent with reported Rap1 targeting of $\mathrm{NuA} 4^{9}$.

Analysis of sonicated ChIP-seq data over Epll-enriched promoters confirmed that Epll binding to these regions was independent of transcription but dependent on the C-terminus of Epl1 (Fig. 2e, inputs in Supplementary Fig. 1j). Moreover, the truncation of Epl1 resulted in a loss of H4K8 acetylation on nucleosomes immediately downstream of TSSs on the Epl1enriched genes (Fig. 2f, inputs in Supplementary Fig. 1k) consistent with $\mathrm{NuA} 4$-dependent acetylation in these regions. Surprisingly, however, the presence of HATs at promoters was insufficient to trigger histone acetylation on its own. Analysis of cells treated with 1,10-pt showed reduced H4K8ac in both wildtype and Epl1 mutants (Fig. 2f), indicating that regardless of the mode of HAT targeting, acetylation remained dependent on transcription. Similar results were observed when analyzing all genes (Fig. 2g, inputs in Supplementary Fig. 1l) or genes lacking divergent transcription (Fig. 2h, inputs in Supplementary Fig. 1m). Collectively, these results confirm the targeting of HATs by transcription activators, but also show that Epl1dependent acetylation of $5^{\prime}$ nucleosomes requires RNAPII activity.

Histone acetyltransferases are regulated post-recruitment. The requirement of transcription for localization of Piccolo to gene bodies implies that Epl1 occupancy should mirror histone $\mathrm{H} 4$ acetylation marks. However, analysis of genes with unidirectional promoters, to avoid the confounding effect of divergent transcription, revealed Epl1 bound throughout gene bodies, while $\mathrm{H} 4$ acetylation was primarily enriched in $5^{\prime}$ regions [Fig. 3a (sonicated ChIP-seq) and b (MNase ChIP-seq), MNase inputs shown in Supplementary Fig. 1n]. Similar results were observed following treatment of cells with TSA (Fig. 3b), and thus the differences between HAT occupancy and acetylated histone levels were not due to HDACs reshaping acetylation patterns. A similar disconnect was observed between the occupancies of the H3-specific HATs, Gcn5, and Sas3, and histone H3 acetylation (Fig. 3c, d, input for $3 \mathrm{~d}$ shown in Supplementary Fig. 1o). Thus, we observe a poor correspondence between HAT localization and histone acetylation, which is consistent with previous suggestions that regulation of HAT activity, following chromatin binding, is a major determinant of histone acetylation genome-wide $21,27,33,39$.

High-resolution mapping of engaged RNAPII by NET-seq, CRAC-seq, and chromatin-bound RNA-seq, shows RNAPII accumulation at the $5^{\prime}$ ends of genes $28,40,41$, which is proposed to represent either slow passage or premature transcription termination of RNAPII in these regions. Consistent with the latter, $5^{\prime}$ accumulation is not observed with PRO-seq ${ }^{42}$, which measures elongation competent RNAPII. The accumulation of histone acetylation at the $5^{\prime}$ ends of genes, together with the transcription-dependence of histone acetylation, suggests that the presence of RNAPII promotes the activity of HATs. In vitro and in silico studies demonstrate that histone tails are tightly associated with DNA, making them poor substrates for $\mathrm{HATs}^{43-46}$, but nucleosome disruption during RNAPII passage could displace tails from DNA, promoting histone acetylation by available HATs. If this hypothesis is correct, then the longer RNAPII spends traversing a nucleosome, the greater is the chance that the histones will be acetylated. To test this, we asked whether nucleosomes that are more likely to impede RNAPII exhibit increased histone acetylation in vivo. To control for total RNAPII levels, annotated gene body nucleosomes ${ }^{47}$ were divided into five bins based on Rpb3 ChIP-seq signal [ $\log _{2}$ (ChIP/input)]. Each bin was further split into quintiles based on the predicted ability to strongly or weakly form nucleosomes ${ }^{48}$. This approach enabled the identification of nucleosomes with similar levels of RNAPII but differing predicted nucleosome occupancies. Data for the middle quintile for RNAPII occupancy are shown in Fig. 3e, with the remaining quintiles shown in Supplementary Figs. 9 and 10. As expected, nucleosome-favoring sequences had increased nucleosome signals, as determined using MNase-seq. Also, despite similar RNAPII occupancies, regions with increased nucleosome occupancies showed enhanced NET-seq, chromatin RNA-seq, and CRAC-seq signals (Fig. 3e and Supplementary Fig. S9), consistent with slower RNAPII passage through these nucleosomes. When normalizing for differing nucleosome occupancy $\left[\log _{2}(\mathrm{ChIP} /\right.$ input)], intrinsically stable nucleosomes were enriched for $\mathrm{H} 4 \mathrm{~K} 12 \mathrm{ac}$, but not Epl1, both under steady-state and TSA-treated conditions (Fig. 3e, Supplementary Figs. 9 and 10). Similar results were observed with $\mathrm{H} 4 \mathrm{~K} 8 \mathrm{ac}$ and $\mathrm{H} 3 \mathrm{~K} 23 \mathrm{ac}$ (Fig. 3e, Supplementary Figs. 9 and 10). Collectively, this work supports a model in which transcription-disrupted nucleosomes are acetylated by available HATs, targeted by either activators or RNAPII.

\section{Discussion}

Previous works suggest that histone acetylation can be both a cause and consequence of transcription and in this study, we sought to define the relative contribution of these pathways to total acetylation. We found that the majority of histone acetylation is dependent on transcription and is targeted to nucleosomes at sites of RNAPII accumulation. To understand the mechanism for targeting this PTM to active genes, we mapped occupancy of Epl1, a component of the NuA4 and Piccolo HAT complexes, in both transcribing and transcription-inhibited cells. The results show that NuA4 is targeted to promoters upstream of transcription, while Piccolo binds gene bodies in a transcriptiondependent manner. At this time, the mechanism for targeting Piccolo to gene bodies is unclear. Although Piccolo contains an H3K4 methyl-reader domain, we found that H3K4 methylation was dispensable for transcription-dependent acetylation ${ }^{27}$. This is reminiscent of previously published work showing that the Yng2 PHD finger is unnecessary for Epl1 targeting ${ }^{27}$. In addition, while ESA1 is required for transcription of approximately half of all yeast genes ${ }^{49}$, set $1 \Delta$ mutants have defects in expression of only a small number of genes ${ }^{13}$, and the role played by H3K4 methylation in gene activation is unclear ${ }^{50}$. Together, these data suggest that another pathway must exist for recruiting Piccolo to transcribed genes. The mammalian homolog of Esa1, Tip60, is proposed to be targeted via R-loops ${ }^{51}$, which has not been reported for NuA4. However, as Esal contains a nucleic acid binding domain ${ }^{52,53}$, this may be a conserved mechanism of HAT recruitment to transcribed regions.

Although histone acetylation requires the interaction of HATs with chromatin, genome-wide HAT occupancy is a poor predictor of this PTM, suggesting additional modes of regulation. In vitro and in silico studies suggest that the histone tails are not exposed to the solution, but rather interact strongly with nucleosomal $\mathrm{DNA}^{43-46}$, which could make them poor substrates for $\mathrm{HATs}^{54}$. However, when transcribing through a nucleosome, 
RNAPII pauses at multiple positions $s^{55-59}$, inducing the formation of partially unwrapped intermediates that likely disrupt the interaction of histone tails with DNA. As such, the accumulation of RNAPII at $5^{\prime}$ genic regions, either due to slow passage or premature termination ${ }^{60}$, increases the opportunity for available HATs to access the histone tails resulting in increased acetylation in these regions. Consistent with this model, we found increased acetylation on nucleosomes predicted to impede RNAPII passage. Histone acetylation is proposed to facilitate transcription by directly modulating histone-DNA contacts or by targeting chromatin remodelers to disrupt nucleosomes ${ }^{61}$. Thus, our research suggests that acetylation is a component of a feedforward loop that maintains the expression of active genes.

\section{Methods}

Cell Culture. FUCCI reporter mESCs ${ }^{62}$ were grown in standard feeder-free conditions in complete mESC media: Dulbecco Modified Eagle's Medium-high glucose, 15\% fetal bovine serum (HyClone Laboratories), $20 \mathrm{mM}$ HEPES, $1 \mathrm{mM} \mathrm{L}$ glutamine, $100 \mathrm{U} / \mathrm{ml}$ penicillin-streptomycin, $1 \mathrm{mM}$ nonessential amino acids, $\sim 10-50 \mathrm{ng} / \mathrm{ml}$ of recombinant LIF, $1 \mathrm{mM}$ sodium pyruvate, and $0.1 \mathrm{mM} \beta-$ mercaptoethanol on $0.2 \%$ type A gelatinized tissue culture plates.

Yeast strains and growth. All strains used in this study were isogenic to S288C and are listed in Supplementary Table 1. Yeast culture and genetic manipulations were performed using standard protocols. Genomic deletions were verified by polymerase chain reaction (PCR) analysis and whole-cell extracts were generated as previously described ${ }^{63}$

Drug treatments. Yeast drug treatments were performed in YPD media at the following concentrations: $400 \mu \mathrm{g} / \mathrm{ml} \mathrm{1,10} \mathrm{phenanthroline} \mathrm{monohydrate} \mathrm{(Sigma}$ 161-0158, dissolved in ethanol), $10 \mu \mathrm{g} / \mathrm{ml}$ thiolutin (Santa Cruz SC-200387, dissolved in DMSO), 1 mM 1-naphthalene acetic acid (Sigma N0640, dissolved in 85\% ethanol), $20 \mu \mathrm{g} / \mathrm{ml}$ doxycycline (Sigma D9891, dissolved in 50\% ethanol), $5 \mu \mathrm{M} \mathrm{1-}$ Naphthyl PP1 (Sigma CAS 221243-82-9, dissolved in DMSO), $25 \mu \mathrm{M}$ trichostatin A (dissolved in DMSO), $10 \mu \mathrm{M}$ a factor (Sigma custom synthesized peptide, WHWLQLKPGQPMY, dissolved in $100 \mathrm{mM}$ sodium acetate, $\mathrm{pH}=5.2)$. mESCs were treated with Actinomycin D at $25 \mu \mathrm{g} / \mathrm{ml}$ (Sigma CAS 50-76-0, dissolved in DMSO).

Immunoblot analysis. Whole-cell lysates or cellular fractions were analyzed by SDS-PAGE using the antibodies listed in the key resource table (Supplementary Table 2) at 1/1000 dilutions. Blots were scanned and fluorescent signal quantified using the Licor Odyssey scanner.

ChIP-seq. Yeast cells, grown to mid-log phase, were arrested in G1 by 3-h treatment with $10 \mu \mathrm{M}$ alpha factor. Cell synchronization was verified by cell "shmooing," as seen under the microscope. For transcription inhibition, cells were treated with $400 \mu \mathrm{g} / \mathrm{ml} 1,10$ phenanthroline monohydrate or $25 \mu \mathrm{M}$ TSA for $15 \mathrm{~m}$. Cells were crosslinked in $1 \%$ formaldehyde for $15 \mathrm{~m}$ and quenched with the addition of liquid glycine to $125 \mathrm{mM}$ for a further $15 \mathrm{~m}$. Cells were lysed by bead beating, and cell lysate was spun down at $15,000 \mathrm{~g}$ for $30 \mathrm{~m}$.

For sonicated ChIP-seq, the pellet was resuspended in lysis buffer $(50 \mathrm{mM}$ HEPES, pH 7.5, $140 \mathrm{mM} \mathrm{NaCl}, 0.5 \mathrm{mM}$ EDTA, 1\% Triton X-100, 0.1\% sodium deoxycholate) and sonicated (Biorupter, Diagenode) to produce an average fragment size of $250 \mathrm{bp}$. The lysate was spun down at $9000 \mathrm{~g}$ for $10 \mathrm{~m}$, and the supernatant was precleared by rotating with Protein $\mathrm{G}$ Dynabeads for $1 \mathrm{~h}$ at $4{ }^{\circ} \mathrm{C}$. Twenty percent of the lysate was reserved for input, and the remaining was incubated with $\mathrm{a}$-HA antibodies overnight at $4{ }^{\circ} \mathrm{C}$

For MNase ChIP-seq, the pellet was resuspended in MNase digestion buffer (0.5 mM spermidine, $1 \mathrm{mM} \beta$-ME, $0.075 \%$ NP-40, $50 \mathrm{mM} \mathrm{NaCl}, 10 \mathrm{mM}$ Tris $\mathrm{pH}$ $7.4,5 \mathrm{mM} \mathrm{MgCl}, 1 \mathrm{mM} \mathrm{CaCl}_{2}$ ). Samples were incubated with 100 units of MNase for $10 \mathrm{~m}$ at $37^{\circ} \mathrm{C}$. Lysates were clarified by centrifugation at $9000 \mathrm{~g}$ for $10 \mathrm{~m}$. To extract insoluble chromatin, pellets were resuspended in $200 \mu \mathrm{l}$ of lysis buffer with $0.2 \%$ SDS and sonicated in a Diagenode Bioruptor at the medium output for $30 \mathrm{~s}$ on and $30 \mathrm{~s}$ off for four cycles, before centrifugation at $9000 \mathrm{~g}$ for $10 \mathrm{~m}$. The second supernatant was pooled with the first, and the buffer composition of the lysate was adjusted to that of the original lysis buffer (50 mM HEPES pH 7.5, $140 \mathrm{mM} \mathrm{NaCl}$, 1 mM EDTA, 2\% Triton X-100, 0.2\% Na-deoxycholate, $1 \times$ Roche protease inhibitor cocktail, $1 \mathrm{mM}$ PMSF). The supernatant was precleared by rotating with Protein G Dynabeads for $1 \mathrm{~h}$ at $4{ }^{\circ} \mathrm{C}, 10 \%$ of the lysate was reserved for input, and immunoprecipitations were performed using $\alpha-\mathrm{HA}, \alpha-\mathrm{H} 3 \mathrm{~K} 23 \mathrm{ac}, \alpha-\mathrm{H} 4 \mathrm{~K} 12 \mathrm{ac}$, or $\alpha-$ H4K8ac antibodies.

Antibody immunoprecipitations were isolated by adding magnetic Protein $\mathrm{G}$ Dynabeads and rotating at $4{ }^{\circ} \mathrm{C}$ for $1 \mathrm{~h}$, and 5 minute washes were performed twice with lysis buffer, twice with high salt buffer (50 mM HEPES pH 7.5, $640 \mathrm{mM} \mathrm{NaCl}$, $1 \mathrm{mM}$ EDTA, $2 \%$ Triton X-100, 0.2\% Na-deoxycholate), twice with $\mathrm{LiCl}$ wash buffer (10 mM Tris-HCl pH 8.0, $250 \mathrm{mM} \mathrm{LiCl}, 0.6 \% \mathrm{NP}-40,0.5 \%$ Na-deoxycholate, $1 \mathrm{mM}$ EDTA), and once with TE. Synthetic spike-in DNA was added to eluates, to aid in quantification. Following proteinase K digestion, DNA was purified by phenol, chloroform, isoamyl alcohol extraction and RNase A treated.

ChIP-seq library preparation. Libraries for paired-end sequencing were constructed using a custom procedure for paired-end sequencing ${ }^{64}$. Briefly, 2-10 ng of ChIP material was end-repaired and A-tailed before being ligated to TruSeq PE adaptors. The adaptor-ligated material was subject to 8-11 rounds of PCR amplification, and an aliquot of each library was run on an Agilent Tape Station to check the size distribution and molarity of the PCR products. Equimolar amounts of indexed, amplified libraries were pooled, and fragments in the 200-600 bp size range were selected on an agarose gel. An aliquot $(1 \mu \mathrm{l})$ of the library pool was run on an Agilent Tape Station to confirm proper size selection. In between each reaction, the material was purified using NucleoMag solid-phase reversible immobilization paramagnetic beads.

Analysis of ChIP-seq data. Adapter sequences were removed from paired-end FASTQ files using cutadapt (version 1.83 - http://cutadapt.readthedocs.io/en/stable/), before aligning to the saccer3 genome using BWA (version 0.7.15-r1140) ${ }^{65}$. For analysis of epll $1_{(1-485)}$ data, reads of chrXII were removed from all data sets, as this chromosome appeared to be unstable in this mutant (1.5× coverage). Coverage tracks represent reads per genome coverage, calculated using the Java Genomics Toolkit (https:/github.com/timpalpant/java-genomics-toolkit) scripts, ngs.BaseAlignCounts and wigmath.Scale. $\log _{2}$ transformed ChIP over input tracks were calculated using the Java Genomics Toolkit and regions without signal in the input were removed to avoid division by 0 . Replicates were pooled for subsequent analysis, and figures were generated in R. FASTQ files from Weiner et al. (2015) were mapped to the saccer3 genome using BWA version 0.7.15-r114065. Reads were extended to $146 \mathrm{bp}$ and read per genome coverage and $\log _{2}$ transformed ChIP over input files were calculated using deepTools version 3.0266. FASTQ files from Steunou et al. (2016) were similarly mapped, but extended to $350 \mathrm{bp}$.

Similar to other groups ${ }^{26,27}$, ChIP-seq datasets from 1,10-pt-treated or Epl1 $1_{(1-}$ 485) cells were normalized to silent regions. The genome was divided into $250 \mathrm{bp}$ bins, bins outside the interquartile range for coverage in the input were discarded, the 100 regions with the lowest Rpb3 signal were defined as silent regions, and these silent regions were used to normalized ChIP-seq datasets for cross-condition comparisons (Supplementary Table 3). We also added synthetic DNA spike-ins to our ChIP eluates and inputs (Supplementary Table 4), but this approach to normalization did not work well for all samples, possibly due to the low coverage of the spike-ins in some samples.

Defining genome annotations. Yeast transcription start and end sites were downloaded from the supplemental files of Chereji et al. ${ }^{67}$. To identify active, nondivergent, yeast promoters, genes in the lowest quintile of NET-seq signal over the first 500 bp downstream of the TSS were designated as non-transcribed. Unidirectional promoters were then defined as transcribed genes with the lowest quintile of NET-seq signal 100-600 bp upstream of the TSS (832 genes). RefSeq mm9 TSSs were downloaded from the UCSC Genome Browser (https://genome. ucsc.edu/). To identify mouse genes with active, non-divergent, promoters, transcribed genes were defined as those with greater than the median PRO-seq signal over $1 \mathrm{~kb}$ downstream of the TSS, and transcribed genes in the lowest quintile of PRO-seq signal upstream of the TSS were designated as having unidirectional promoters (3035 genes).

For transcribed nucleosomes classified by Rpb3 change upon 1,10-pt treatment (Supplementary Figs. 3B and 5B), genome-wide nucleosome positions ${ }^{47}$ with Rpb3 signal greater than the median were classified as transcribed. Nucleosomes, where Rpb3 changed by less than $10 \%$, were classified as "Rpb3 stable", while those decreasing by at least $3 \times$ were classified as "Rpb3 lost". Boxplots represent the first to third quartiles, with whiskers extending to 1.5 times the interquartile range or to the extreme of the data. Notches are equal to $\pm 1 / 58$ IQR /sqrt $(n)$, giving an approximation of the $95 \%$ confidence interval for the difference in 2 medians.

To find promoter peaks of Epll, the Epll MNase ChIP-seq was compared to its input within the NDR for each gene ${ }^{67}$. Within each NDR, a smoothing spline was fit to the IP minus input signal (RPGC) and the peak position was selected. Peak positions with an IP minus input greater than 0.5 RPGC in the Epll ChIP-seq but not in the untagged control were selected as Epll peaks. NDRs in close proximity to tRNA genes or centromeres were removed from further analysis due to the binding of Epl1 to these elements.

For motif analysis, Epll peaks were compared to 1958 NDR regions depleted for Epl1 binding (maximum IP minus input less than $0.1 \mathrm{RPGC}$ ). The $500 \mathrm{bp}$ regions around peak centers were then inputted into the MEME-ChIP Differential Enrichment algorithm ${ }^{68}$ to find enriched motifs from the JASPAR nonredundant core fungi motifs ${ }^{69}$. For the top two hits, Rap1 and Aft2, CentriMo ${ }^{70}$ was used to plot the distance from the best motif site to the Epll peak center and the motif probabilities around the best motif site for the regions containing target motifs.

Generating heatmaps and metaplots. Metaplot matrices centered on TSSs were generated using the sitepro script from the CEAS package $1.0 .2^{71}$ and matrices 
aligned to other features were produced using the visualization.MatrixAligner script from the Java Genomics Toolkit. Heatmaps were generated using deepTools $^{66}$, and for 2D heatmaps, plot2DO (version 1) was used ${ }^{72}$.

Reporting summary. Further information on research design is available in the Nature Research Reporting Summary linked to this article.

\section{Data availability}

Data generated for this manuscript were deposited in the NCBI Gene Expression Omnibus under the accession code "GSE110287". Published datasets analyzed for this paper include "SRP132377" (S. cerevisiae RNAPII ChIP-seq $\left.\pm 1,10-\mathrm{pt}^{73}\right)$, "SRP095935" (S. cerevisiae H3K23ac \pm TSA and Sas3 ChIP-seq ${ }^{33}$ ), "SRP048526” (S. cerevisiae histone PTM MNase ChIP-seq ${ }^{47}$ ), “GSE68484” (S. cerevisiae NET-seq $\left.{ }^{41}\right)$, “GSE68484” (S. cerevisiae chromatin RNA-seq $\left.{ }^{41}\right)$, “GSE69676” (S. cerevisiae CRAC-seq $\left.{ }^{40}\right)$, http://sgdarchive.yeastgenome.org/published_datasets/MacIsaac_2006_PMID_16522208/ track_files/MacIsaac_2006_ChIP_chip_TFBSs_V64.gff3 (S. cerevisiae TFBS ${ }^{38}$ ), "SRP070154" (S. cerevisiae Epl1 and H4K8ac ChIP-chip ${ }^{27}$ ), "GSE36600" (S. cerevisiae Gcn5 and H3K18ac ChIP-chip ${ }^{32}$ ), http://sgd-archive.yeastgenome.org/ published_datasets/Kaplan_2009_PMID_19092803/track_files/

Kaplan_2009_predicted_average_nucleosome_occupancy_V64.wig (S. cerevisiae Predicted nucleosome occupancy ${ }^{48}$ ), https://www.ncbi.nlm.nih.gov/pmc/articles/ PMC5807854/bin/13059_2018_1398_MOESM2_ESM.xlsx (S. cerevisiae TSS and PAS annotations ${ }^{67}$ ), https://www.ncbi.nlm.nih.gov/pmc/articles/PMC4405355/bin/mmc3.csv (S. cerevisiae genome-wide nucleosome positions ${ }^{47}$ ), "GSE130691" (M. musculus mESC PRO-seq ${ }^{74}$ ), “GSE31039” (M. musculus mESC H3K9ac and H3K27ac ChIP-seq, Mouse ENCODE epigenomic data). All relevant data supporting the key findings of this study are available within the article and its Supplementary Information files or from the corresponding author upon reasonable request. Source data for Fig. 1a, d, and Epll peak midpoints are provided in the Source Data file. A reporting summary for this Article is available as a Supplementary Information file. Source data are provided with this paper.

\section{Code availability}

The following software packages were used in this study: cutadapt (version 1.83-http:// cutadapt.readthedocs.io/en/stable/), BWA (version 0.7.15-r1140) ${ }^{65}$, Java Genomics Toolkit (https://github.com/timpalpant/java-genomics-toolkit), deepTools version $3.02^{66}$. CEAS package version 1.0.2 $2^{71}$, plot2DO ${ }^{72}$, the MEME-ChIP Differential Enrichment algorithm $^{68}$, and CentriMo ${ }^{70}$.

Received: 23 September 2019; Accepted: 8 December 2020; Published online: 11 January 2021

\section{References}

1. Van Holde, K. E. Chromatin. (Springer-Verlag, 1989).

2. Rando, O. J. Global patterns of histone modifications. Curr. Opin. Genet. Dev. 17, 94-99 (2007).

3. Friis, R. M. \& Schultz, M. C. Untargeted tail acetylation of histones in chromatin: lessons from yeast. Biochem. Cell Biol. 87, 107-116 (2009).

4. Lee, K. K. \& Workman, J. L. Histone acetyltransferase complexes: one size doesn't fit all. Nat. Rev. Mol. Cell Biol. 8, 284-295 (2007).

5. Dion, M. F., Altschuler, S. J., Wu, L. F. \& Rando, O. J. Genomic characterization reveals a simple histone H4 acetylation code. Proc. Natl Acad. Sci. USA 102, 5501-5506 (2005).

6. Yang, X. J. \& Seto, E. The Rpd3/Hdal family of lysine deacetylases: from bacteria and yeast to mice and men. Nat. Rev. Mol. Cell Biol. 9, 206-218 (2008).

7. Brown, C. E. et al. Recruitment of HAT complexes by direct activator interactions with the ATM-related Tral subunit. Science 292, 2333-2337 (2001).

8. Reeves, W. M. \& Hahn, S. Targets of the Gal4 transcription activator in functional transcription complexes. Mol. Cell. Biol. 25, $9092-9102$ (2005)

9. Knutson, B. A. \& Hahn, S. Domains of Tral important for activator recruitment and transcription coactivator functions of SAGA and NuA4 complexes. Mol. Cell. Biol. 31, 818-831 (2011).

10. Baptista, T. et al. SAGA is a general cofactor for RNA polymerase II transcription. Mol. Cell https://doi.org/10.1016/j.molcel.2017.08.016 (2017).

11. Reid, J. L., Iyer, V. R., Brown, P. O. \& Struhl, K. Coordinate regulation of yeast ribosomal protein genes is associated with targeted recruitment of Esal histone acetylase. Mol. Cell 6, 1297-1307 (2000).

12. Ginsburg, D. S., Govind, C. K. \& Hinnebusch, A. G. NuA4 lysine acetyltransferase Esal is targeted to coding regions and stimulates transcription elongation with Gcn5. Mol. Cell. Biol. 29, 6473-6487 (2009).

13. Lenstra, T. L. et al. The specificity and topology of chromatin interaction pathways in yeast. Mol. Cell 42, 536-549 (2011).
14. Downey, M. \& Baetz, K. Building a KATalogue of acetyllysine targeting and function. Brief Funct. Genomics 15, 109-118 (2016).

15. Martin, D. G. et al. The Ynglp plant homeodomain finger is a methyl-histone binding module that recognizes lysine 4-methylated histone H3. Mol. Cell. Biol. 26, 7871-7879 (2006).

16. Shi, X. et al. ING2 PHD domain links histone $\mathrm{H} 3$ lysine 4 methylation to active gene repression. Nature 442, 96-99 (2006).

17. Taverna, S. D. et al. Yng1 PHD finger binding to $\mathrm{H} 3$ trimethylated at $\mathrm{K} 4$ promotes NuA3 HAT activity at K14 of $\mathrm{H} 3$ and transcription at a subset of targeted ORFs. Mol. Cell 24, 785-796 (2006).

18. Bian, C. et al. Sgf29 binds histone H3K4me2/3 and is required for SAGA complex recruitment and histone $\mathrm{H} 3$ acetylation. EMBO J. 30, 2829-2842 (2011).

19. Govind, C. K., Zhang, F., Qiu, H., Hofmeyer, K. \& Hinnebusch, A. G. Gen5 promotes acetylation, eviction, and methylation of nucleosomes in transcribed coding regions. Mol. Cell 25, 31-42 (2007).

20. Venkatesh, S. et al. Set 2 methylation of histone H3 lysine 36 suppresses histone exchange on transcribed genes. Nature 489, 452-455 (2012).

21. Bose, D. A. et al. RNA binding to CBP stimulates histone acetylation and transcription. Cell 168, 135-149 (2017). e122.

22. Durant, M. \& Pugh, B. F. Genome-wide relationships between TAF1 and histone acetyltransferases in Saccharomyces cerevisiae. Mol. Cell. Biol. 26, 2791-2802 (2006).

23. Sun, M. et al. Global analysis of eukaryotic mRNA degradation reveals Xrn1dependent buffering of transcript levels. Mol. Cell 52, 52-62 (2013).

24. Kim, T. S. et al. RNA polymerase mapping during stress responses reveals widespread nonproductive transcription in yeast. Genome Biol. 11, R75 (2010).

25. Sobell, H. M. Actinomycin and DNA transcription. Proc. Natl Acad. Sci. USA 82, 5328-5331 (1985).

26. Petrenko, N., Jin, Y., Wong, K. H. \& Struhl, K. Mediator undergoes a compositional change during transcriptional activation. Mol. Cell 64, 443-454 (2016).

27. Steunou, A. L. et al. Combined action of histone reader modules regulates $\mathrm{NuA} 4$ local acetyltransferase function but not its recruitment on the genome. Mol. Cell. Biol. https://doi.org/10.1128/MCB.00112-16 (2016).

28. Churchman, L. S. \& Weissman, J. S. Nascent transcript sequencing visualizes transcription at nucleotide resolution. Nature 469, 368-373 (2011).

29. Duttke, S. H. C. et al. Human promoters are intrinsically directional. Mol. Cell 57, 674-684 (2015).

30. Scruggs, B. S. et al. Bidirectional transcription arises from two distinct hubs of transcription factor binding and active chromatin. Mol. Cell 58, 1101-1112 (2015).

31. Boudreault, A. A. et al. Yeast enhancer of polycomb defines global Esa1dependent acetylation of chromatin. Genes Dev. 17, 1415-1428 (2003).

32. Xue-Franzen, Y., Henriksson, J., Burglin, T. R. \& Wright, A. P. Distinct roles of the Gcn5 histone acetyltransferase revealed during transient stress-induced reprogramming of the genome. BMC Genomics 14, 479 (2013).

33. Martin, B. J. et al. Histone H3K4 and H3K36 methylation independently recruit the $\mathrm{NuA} 3$ histone acetyltransferase in Saccharomyces cerevisiae. Genetics 205, 1113-1123 (2017).

34. Shi, X. et al. Proteome-wide analysis in Saccharomyces cerevisiae identifies several PHD fingers as novel direct and selective binding modules of histone H3 methylated at either lysine 4 or lysine 36. J. Biol. Chem. 282, 2450-2455 (2007).

35. Ginsburg, D. S. et al. NuA4 links methylation of histone $\mathrm{H} 3$ lysines 4 and 36 to acetylation of histones H4 and H3. J. Biol. Chem. 289, 32656-32670 (2014).

36. Sathianathan, A. et al. The Eaf $3 / 5 / 7$ subcomplex stimulates NuA4 interaction with methylated histone H3 Lys-36 and RNA polymerase II. J. Biol. Chem. 291, 21195-21207 (2016).

37. Chereji, R. V., Ocampo, J. \& Clark, D. J. MNase-sensitive complexes in yeast: nucleosomes and non-histone barriers. Mol. Cell 65, 565-577 (2017). e563.

38. MacIsaac, K. D. et al. An improved map of conserved regulatory sites for Saccharomyces cerevisiae. BMC Bioinform. 7, 113 (2006).

39. Rada-Iglesias, A. et al. A unique chromatin signature uncovers early developmental enhancers in humans. Nature 470, 279-283 (2011).

40. Milligan, L. et al. Strand-specific, high-resolution mapping of modified RNA polymerase II. Mol. Syst. Biol. 12, 874 (2016).

41. Harlen, K. M. et al. Comprehensive RNA polymerase II interactomes reveal distinct and varied roles for each phospho-CTD residue. Cell Rep. 15, 2147-2158 (2016).

42. Booth, G. T., Wang, I. X., Cheung, V. G. \& Lis, J. T. Divergence of a conserved elongation factor and transcription regulation in budding and fission yeast. Genome Res. 26, 799-811 (2016).

43. Li, Z. \& Kono, H. Distinct roles of histone $\mathrm{H} 3$ and $\mathrm{H} 2 \mathrm{~A}$ tails in nucleosome stability. Sci. Rep. 6, 31437 (2016).

44. Ikebe, J., Sakuraba, S. \& Kono, H. H3 histone tail conformation within the nucleosome and the impact of K14 acetylation studied using enhanced sampling simulation. PLoS Comput. Biol. 12, e1004788 (2016). 
45. Shaytan, A. K. et al. Coupling between histone conformations and DNA geometry in nucleosomes on a microsecond timescale: atomistic insights into nucleosome functions. J. Mol. Biol. https://doi.org/10.1016/j.jmb.2015.12.004 (2015).

46. Gatchalian, J. et al. Accessibility of the histone $\mathrm{H} 3$ tail in the nucleosome for binding of paired readers. Nat. Commun. 8, 1489 (2017).

47. Weiner, A. et al. High-resolution chromatin dynamics during a yeast stress response. Mol. cell 58, 371-386 (2015).

48. Kaplan, N. et al. The DNA-encoded nucleosome organization of a eukaryotic genome. Nature 458, 362-366 (2009).

49. Bruzzone, M. J., Grunberg, S., Kubik, S., Zentner, G. E. \& Shore, D. Distinct patterns of histone acetyltransferase and mediator deployment at yeast protein-coding genes. Genes Dev. https://doi.org/10.1101/gad.312173.118 (2018).

50. Howe, F. S., Fischl, H., Murray, S. C. \& Mellor, J. Is H3K4me3 instructive for transcription activation? Bioessays 39, 1-12 (2017).

51. Chen, P. B., Chen, H. V., Acharya, D., Rando, O. J. \& Fazzio, T. G. R loops regulate promoter-proximal chromatin architecture and cellular differentiation. Nat. Struct. Mol. Biol. 22, 999-1007 (2015).

52. Huang, J. \& Tan, S. Piccolo NuA4-catalyzed acetylation of nucleosomal histones: critical roles of an Esal Tudor/chromo barrel loop and an Epll enhancer of polycomb A (EPcA) basic region. Mol. Cell. Biol. 33, 159-169 (2013).

53. Shimojo, H. et al. Novel structural and functional mode of a knot essential for RNA binding activity of the Esal presumed chromodomain. J. Mol. Biol. 378, 987-1001 (2008)

54. Stutzer, A. et al. Modulations of DNA contacts by linker histones and posttranslational modifications determine the mobility and modifiability of nucleosomal H3 tails. Mol. Cell https://doi.org/10.1016/j.molcel.2015.12.015 (2016).

55. Kulaeva, O. I., Hsieh, F. K., Chang, H. W., Luse, D. S. \& Studitsky, V. M. Mechanism of transcription through a nucleosome by RNA polymerase II. Biochim. Biophys. Acta 1829, 76-83 (2013).

56. Bintu, L. et al. Nucleosomal elements that control the topography of the barrier to transcription. Cell 151, 738-749 (2012).

57. Ehara, H. et al. Structural insight into nucleosome transcription by RNA polymerase II with elongation factors. Science https://doi.org/10.1126/science. aav8912 (2019)

58. Farnung, L., Vos, S. M. \& Cramer, P. Structure of transcribing RNA polymerase II-nucleosome complex. Nat. Commun. 9, 5432 (2018).

59. Kujirai, T. et al. Structural basis of the nucleosome transition during RNA polymerase II passage. Science https://doi.org/10.1126/science.aau9904 (2018).

60. Ehrensberger, A. H., Kelly, G. P. \& Svejstrup, J. Q. Mechanistic interpretation of promoter-proximal peaks and RNAPII density maps. Cell 154, 713-715 (2013).

61. Choi, J. K. \& Howe, L. J. Histone acetylation: truth of consequences? Biochem. Cell Biol. 87, 139-150 (2009).

62. Chen, C. C. L. et al. H3S10ph broadly marks early-replicating domains in interphase ESCs and shows reciprocal antagonism with $\mathrm{H} 3 \mathrm{~K} 9 \mathrm{me2}$. Genome Res. 28, 37-51 (2018).

63. Kushnirov, V. V. Rapid and reliable protein extraction from yeast. Yeast 16, 857-860 (2000).

64. Maltby, V. E. et al. Histone H3K4 demethylation is negatively regulated by histone H3 acetylation in Saccharomyces cerevisiae. Proc. Natl Acad. Sci. USA 109, 18505-18510 (2012).

65. Li, H. \& Durbin, R. Fast and accurate long-read alignment with BurrowsWheeler transform. Bioinformatics 26, 589-595 (2010).

66. Ramirez, F. et al. deepTools2: a next generation web server for deepsequencing data analysis. Nucleic Acids Res. 44, W160-W165 (2016).

67. Chereji, R. V., Ramachandran, S., Bryson, T. D. \& Henikoff, S. Precise genome-wide mapping of single nucleosomes and linkers in vivo. Genome Biol. 19, 19 (2018).

68. Ma, W., Noble, W. S. \& Bailey, T. L. Motif-based analysis of large nucleotide data sets using MEME-ChIP. Nat. Protoc. 9, 1428-1450 (2014).

69. Khan, A. et al. JASPAR 2018: update of the open-access database of transcription factor binding profiles and its web framework. Nucleic Acids Res. 46, D260-D266 (2018)
70. Bailey, T. L. \& Machanick, P. Inferring direct DNA binding from ChIP-seq Nucleic Acids Res. 40, e128 (2012).

71. Shin, H., Liu, T., Manrai, A. K. \& Liu, X. S. CEAS: cis-regulatory element annotation system. Bioinformatics 25, 2605-2606 (2009).

72. Beati, P. \& Chereji, R. V. Creating 2D occupancy plots using plot2DO. Methods Mol. Biol. 2117, 93-108 (2020).

73. Martin, B. J. E., Chruscicki, A. T. \& Howe, L. J. Transcription promotes the interaction of the FAcilitates Chromatin Transactions (FACT) complex with nucleosomes in Saccharomyces cerevisiae. Genetics 210, 869-881 (2018).

74. Etchegaray, J. P. et al. The histone deacetylase SIRT6 restrains transcription elongation via promoter-proximal pausing. Mol. Cell 75, 683-699 (2019). e687.

75. Zunder, R. M., Antczak, A. J., Berger, J. M. \& Rine, J. Two surfaces on the histone chaperone Rtt106 mediate histone binding, replication, and silencing. Proc. Natl Acad. Sci. USA 109, E144-E153 (2012).

\section{Acknowledgements}

Support for this work was provided by grants to L.J.H. and M.C.L. from the Canadian Institutes of Health Research (PJT-162253) and Natural Sciences and Engineering Research Council (RGPIN-2018-04907). B.J.E.M. was supported by a fellowship from the Natural Sciences and Engineering Research Council. We are grateful to Steven Hahn, Michael Kobor, and Hiroshi Kimura for providing plasmids and antibodies.

\section{Author contributions}

Conception: L.J.H. and B.J.E.M.; acquisition: B.J.E.M., J.B., A.K., K.N., and J.L.; analysis: L.J.H. and B.J.E.M.; drafting, B.J.E.M.; revision, B.J.E.M., L.J.H., J.B., and M.C.L.

\section{Competing interests}

The authors declare no competing interests.

\section{Additional information}

Supplementary information is available for this paper at https://doi.org/10.1038/s41467020-20543-z.

Correspondence and requests for materials should be addressed to L.J.H.

Peer review information Nature Communications thanks Pradeepa Madapura and the other, anonymous, reviewer(s) for their contribution to the peer review of this work. Peer reviewer reports are available.

Reprints and permission information is available at http://www.nature.com/reprints

Publisher's note Springer Nature remains neutral with regard to jurisdictional claims in published maps and institutional affiliations.

Open Access This article is licensed under a Creative Commons Attribution 4.0 International License, which permits use, sharing, adaptation, distribution and reproduction in any medium or format, as long as you give appropriate credit to the original author(s) and the source, provide a link to the Creative Commons license, and indicate if changes were made. The images or other third party material in this article are included in the article's Creative Commons license, unless indicated otherwise in a credit line to the material. If material is not included in the article's Creative Commons license and your intended use is not permitted by statutory regulation or exceeds the permitted use, you will need to obtain permission directly from the copyright holder. To view a copy of this license, visit http://creativecommons.org/ licenses/by/4.0/.

(C) The Author(s) 2021 